\title{
Cardiac Imaging and Management of Prosthetic Valve Candida Parapsilosis Endocarditis
}

\author{
Arjun C. Khadilkar ${ }^{1}$, Jesus Diaz Vera ${ }^{1}$, Juan Enciso ${ }^{1}$, Paula Hernandez Burgos ${ }^{2}$ \\ 1. Internal Medicine, University of South Florida, Tampa, USA $\quad$ 2. Cardiology, University of South Florida, Tampa, USA
}

Corresponding author: Arjun C. Khadilkar, akhadilkar18@gmail.com

\begin{abstract}
Fungal infective endocarditis is a rare and serious form of endocarditis associated with severe morbidity and mortality. The greatest propensity for infection can be found in patients with implanted prosthetic valves, implanted cardiac devices, and intravenous drug use. We present a case of a 45 -year-old male with a prior bioprosthetic mitral valve who was diagnosed with Candida parapsilosis endocarditis. Computed tomography imaging of the abdomen was significant for splenic infarcts, and transesophageal echocardiography demonstrated a $1.23 \mathrm{~cm} \times 0.55 \mathrm{~cm}$ lesion and $1.02 \mathrm{~cm}$ x $0.545 \mathrm{~cm}$ lesion on the bioprosthetic valve. The patient was subsequently treated with Amphotericin B and life-long Fluconazole. This case highlights the imaging findings and treatment of a rare disease process.
\end{abstract}

Review began 05/22/2021 Review ended 06/24/2021 Published 07/01/2021

\section{○ Copyright 2021}

Khadilkar et al. This is an open access article distributed under the terms of the Creative Commons Attribution License CC-BY 4.0., which permits unrestricted use, distribution, and reproduction in any medium, provided the original author and source are credited.
Categories: Cardiology, Internal Medicine, Infectious Disease

Keywords: candida endocarditis, prosthetic heart valve, fungal, splenic infarcts, transthoracic and transesophageal echocardiography

\section{Introduction}

Fungal infective endocarditis (IE) is a rare and serious form of IE with mortality rates up to 50\% [1]. Typical risk factors include prosthetic valve implantation, cardiac implantation devices, and intravenous drug use [1]. In general, the most common etiologic causes of fungal IE are the Candida and Aspergillus species [1]. A six-year case review examining 12 separate cases of Candida IE found the most common organisms were Candida parapsilosis $(\mathrm{n}=8,67 \%)$, Candida glabrata $(\mathrm{n}=3,25 \%)$, and Candida albicans $(\mathrm{n}=1$, 8\%) [2]. Current guidelines by the Infectious Diseases Society of America Candidiasis and American Heart Association Endocarditis recommend treatment of Candida IE with either Amphotericin B with or without Flucytosine or high-dose echinocandin therapy, followed by life-long maintenance therapy with an oral azole [2]. However, treatment failure with medical management alone is common because Candida species have adapted survival strategies including the formation of biofilms on native and prosthetic heart valves, which can lead to poor antifungal activity [1]. Therefore, it is imperative that the treatment of native valve endocarditis includes a potential surgical option, especially in patients with prosthetic valves since early intervention has been proven beneficial [1]. Despite combined medical and surgical treatment, fungal IE has a poor overall prognosis, and there is an imperative need for aggressive risk factor management.

We present a case of a 45-year-old male with Candida parapsilosis IE in the setting of a bioprosthetic mitral valve and highlight the computed tomography (CT) abdomen and pelvis, transthoracic echocardiography (TTE), and transesophageal echocardiography (TEE) image findings associated with this devastating disease process.

\section{Case Presentation}

A 45-year-old male with a history of intravenous drug use, liver cirrhosis secondary to hepatitis C, recent mitral valve endocarditis with a bioprosthetic mitral valve in 2019, gastroesophageal reflux disease (GERD), and pancytopenia presented with four months of progressive abdominal distention. The patient lost his insurance during the coronavirus-19 pandemic and was not following up with routine healthcare. His abdominal distention was causing decreased appetite and early satiety and led to a 40-pound unintentional weight loss. The patient denied associated symptoms of nausea, vomiting, or diarrhea and felt that his symptoms of GERD were well-controlled with daily pantoprazole. In addition, the patient was having intermittent fevers for two weeks and shortness of breath for three days prior to admission.

On admission, the patient was febrile with a temperature of 101 degrees Fahrenheit, normotensive with blood pressure 116/77 millimeters of mercury (mmHg), tachycardia with a heart rate of 114 beats per minute, and saturating $98 \%$ on room air. Initial labs are listed in Table 1. 


\section{Cureus}

\section{Name of Lab}

High-sensitivity C-reactive protein

Aspartate transaminase (AST)

Total bilirubin

Sedimentation rate

Troponin

White blood cell count

Hemoglobin

Platelets

Hepatitis C viral load

\section{Patient Value}

$6.75 \mathrm{mg} / \mathrm{dL}$

$41 \mathrm{U} / \mathrm{L}$

$1.9 \mathrm{mg} / \mathrm{dL}$

$41 \mathrm{~mm} / \mathrm{hr}$

$<0.010 \mathrm{ng} / \mathrm{mL}$

$5.26 \times 10^{3} / \mathrm{uL}$

$9.7 \mathrm{~g} / \mathrm{dL}$

$45 \times 10^{3} / \mathrm{uL}$

$<10 \mathrm{IU} / \mathrm{mL}$
Normal Range

$0.01-0.05 \mathrm{mg} / \mathrm{dL}$

5-34 U/L

$0.1-1.2 \mathrm{mg} / \mathrm{dL}$

$0-15 \mathrm{~mm} / \mathrm{hr}$

$0.000-0.028 \mathrm{mg} / \mathrm{dL}$

$4.6-10.2 \times 10^{3} / \mathrm{uL}$

$14.1-18.1 \mathrm{~g} / \mathrm{dL}$

$142-424 \times 10^{3} / \mathrm{uL}$

$<10 \mathrm{IU} / \mathrm{mL}$

TABLE 1: Patient's labs on presentation

CT abdomen and pelvis were remarkable for splenomegaly with new acute to subacute splenic infarcts, the presence of cirrhosis with moderate volume ascites, and portal enteropathy (Figure 1). Chest x-ray showed worsening bilateral airspace disease with perihilar distribution, and CT angiography of the pulmonary arteries was negative for acute central pulmonary embolus. Blood cultures were ordered, and the patient was started on empiric Cefepime. 


\section{Cureus}

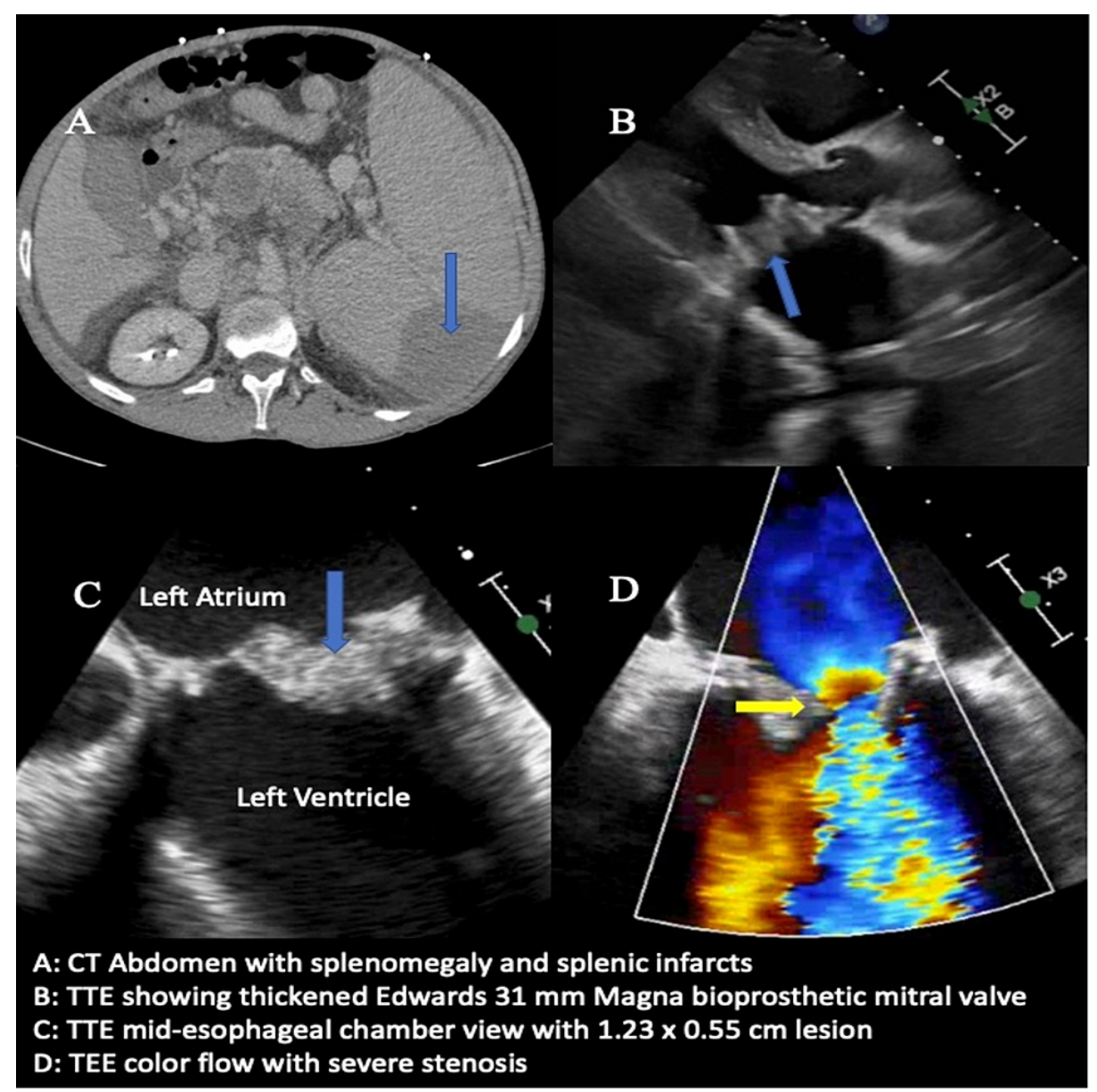

FIGURE 1: Computed tomography of the abdomen, transthoracic echocardiography, and transesophageal echocardiography findings

D: Doppler findings; mitral mean velocity: $2.7 \mathrm{~m} / \mathrm{sec}$; mitral mean gradient: $17 \mathrm{~mm} \mathrm{Hg}$ (severe > $10 \mathrm{~mm} \mathrm{Hg}$ )

Thoracentesis was performed to relieve his respiratory symptoms. Approximately 1200 milliliters of cloudy amber fluid was drained out, which had moderate white blood cells without the growth of any organisms. TTE showed a preserved left ventricular ejection fraction of 55\%-60\% and a 31-millimeter (mm) Edwards bioprosthetic valve with severely thickened leaflets and restricted motion. TTE was unequivocal in completely excluding any vegetation or mass on the bioprosthetic valve. The mean mitral valve gradient was elevated at $28 \mathrm{~mm} \mathrm{Hg}$ (normal < $13 \mathrm{~mm} \mathrm{Hg}$ ), and the pulmonary artery pressure was $74 \mathrm{~mm} \mathrm{Hg}$ (normal 18$25 \mathrm{~mm} \mathrm{Hg}$ ) (Figure 1). Initial blood cultures grew in Candida parapsilosis, and the patient was started on intravenous (IV) Micafungin $100 \mathrm{mg}$ daily. TEE study performed subsequently during the same anesthesia showed a $1.23 \mathrm{~cm} \times 0.55 \mathrm{~cm}$ lesion and $1.02 \mathrm{~cm}$ x $0.55 \mathrm{~cm}$ lesion on the Edwards $31 \mathrm{~mm}$ Magna bioprosthetic mitral valve consistent with vegetation versus thrombus (Figure 2). The patient was deemed not a surgical candidate due to concomitant liver cirrhosis, esophageal varices, ascites, and elevated model for end-stage liver disease (MELD) score of 13-15. He was temporarily treated with IV Amphotericin B $200 \mathrm{mg}$ daily for seven days and was ultimately discharged with six weeks of IV Fluconazole $400 \mathrm{mg}$ daily followed by lifelong oral Fluconazole 200 mg daily. 


\section{Cureus}

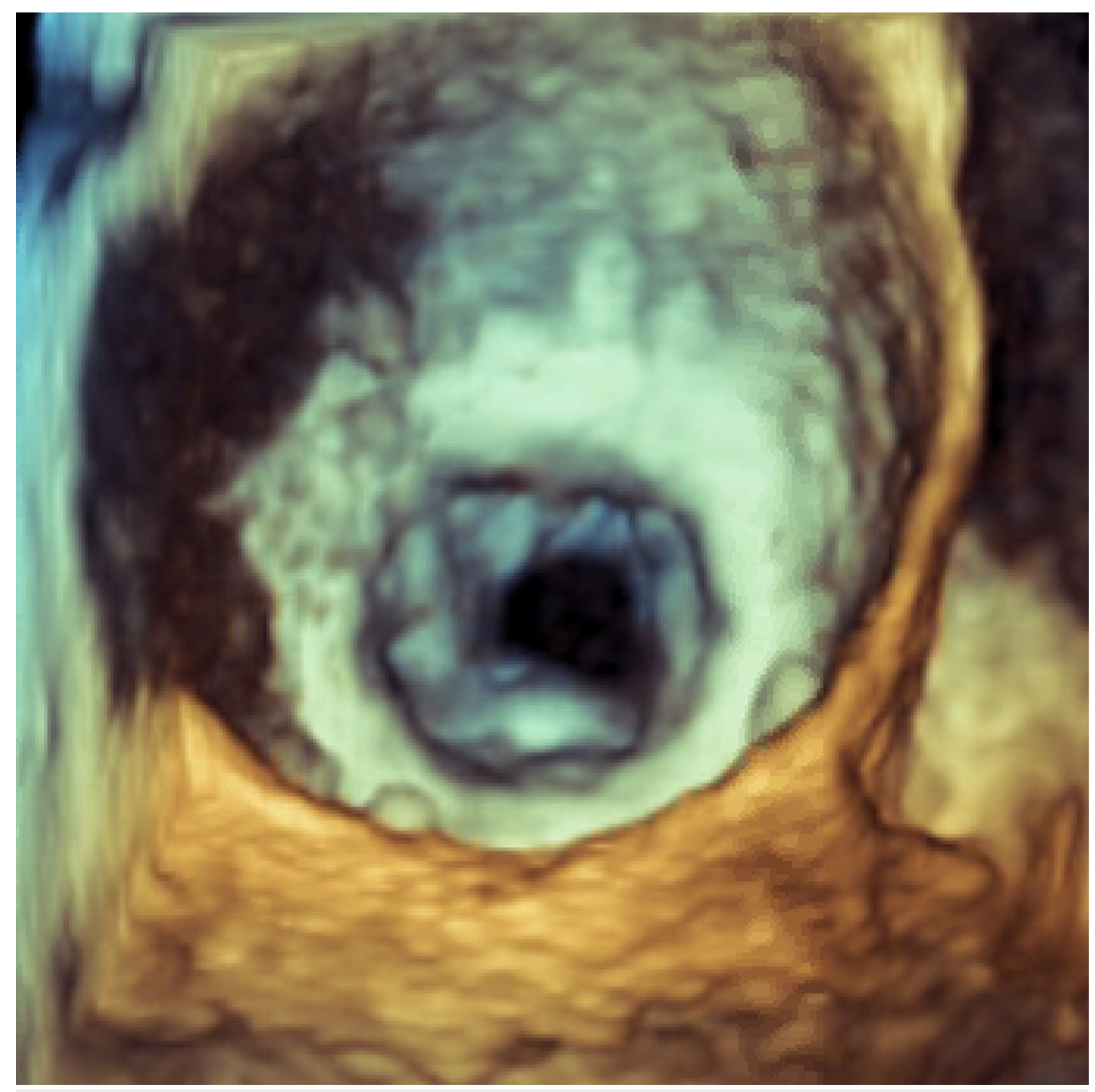

\section{FIGURE 2: Three-dimensional transesophageal echocardiogram of surgeon's view during diastole}

Three-dimensional planimeter valve area: $0.77-0.87 \mathrm{~cm}^{2}\left(\right.$ Severe $\left.<1.0 \mathrm{~cm}^{2}\right)$.

\section{Discussion}

Fungal prosthetic valve IE is a rare presentation of IE affecting people worldwide. In a prior literature review, a total of 152 cases were identified from 1995 to 2000, with intravenous injection drug use as an identified risk factor in only $4.1 \%$ of cases [3]. Fungal IE is associated with high incidences of severe morbidity and mortality, ranging from overall morbidity of $67 \%$ and six-month mortality risk of $37 \%[4,5]$. The rarity of published reports and the medical guidelines for the appropriate choice and duration of antifungal therapy has been limited. Of those case reports published, medical management with liposomal Amphotericin B (LAmB) $5 \mathrm{mg} / \mathrm{kg} /$ day (300 mg/day) and Flucytosine $150 \mathrm{mg} / \mathrm{kg} / \mathrm{day}(9 \mathrm{~g} /$ day) as part of an initial treatment after positive blood cultures for Candida is the suggested intervention [6]. The latest Infectious Diseases Society of America (IDSA) from 2016 provides guidelines regarding the treatment of Candida infections with relationship to infected pacemakers, implantable cardiac defibrillators, ventricular assist devices, along with the treatment of native valve and prosthetic valve IE [7]. These guidelines recommend LAmB of 3-5 milligrams (mg) per kilogram (kg), with or without Flucytosine $25 \mathrm{mg} / \mathrm{kg}$ four times daily, or high-dose echinocandin therapy (Caspofungin $150 \mathrm{mg}$ daily, Micafungin $150 \mathrm{mg}$ daily, or Anidulafungin $200 \mathrm{mg}$ daily) [7]. In addition, for patients unable to undergo valve replacement, there is a strong recommendation for chronic long-term suppression with Fluconazole $400-800 \mathrm{mg}$ daily [7]. Our patient continues to follow up with cardiology as an outpatient. The most recent echocardiogram completed in March 2021 showed decreased visualization of the bioprosthetic valve vegetation with a stable mitral valve peak velocity of 2.06 $\mathrm{m} / \mathrm{s}$.

Recent clinical studies have provided additional insight into Candida IE. One study published by Rivosky et al. monitored 46 patients with prosthetic valve IE who were treated with LAmB versus echinocandin-based induction therapy over a median of nine months. The patients who received only LAmB has a better sixmonth survival rate compared to patients who received only echinocandins (adjusted odds ratio: 13.52, 95\% confidence interval 1.03-838.10) [4]. In addition, 21 patients received long-term maintenance therapy with Fluconazole, with an average of 13 months, and demonstrated minimal adverse effects [4]. Also, the 19 
patients who underwent a cardiac surgical procedure did not have improved survival outcomes over a sixmonth period as compared to the group treated with medical management [4]. These results offer some contradiction to the Infectious Disease of America and European Society of Clinical Microbiology and Infectious Diseases guidelines, which recommend early surgical intervention for all patients with prosthetic IE $[5,8]$. Despite the poor statistical power with a small sample size, this study provides an insight into medical management as an alternative to surgical therapy.

\section{Conclusions}

Candida IE is a rare pathological process with a high propensity to involve and compromise implanted prosthetic valves and implanted cardiac devices. Individuals with a history of drug use are among the greatest risk in the general population. In our case, the patient had a previous history of bioprosthetic mitral valve replacement and was found to have recurrent IE on his bioprosthetic mitral valve. Ultimately, the patient was not a surgical candidate and was treated with Amphotericin B and long-term Fluconazole. This case focuses on the presentation of Candida parapsilosis IE with subsequent imaging findings of this rare disease. In addition, this case highlights the importance of reviewing current clinical guidelines and providing appropriate medical management.

\section{Additional Information \\ Disclosures}

Human subjects: Consent was obtained or waived by all participants in this study. Conflicts of interest: In compliance with the ICMJE uniform disclosure form, all authors declare the following: Payment/services info: All authors have declared that no financial support was received from any organization for the submitted work. Financial relationships: All authors have declared that they have no financial relationships at present or within the previous three years with any organizations that might have an interest in the submitted work. Other relationships: All authors have declared that there are no other relationships or activities that could appear to have influenced the submitted work.

\section{References}

1. Yuan SM: Fungal endocarditis. Braz J Cardiovasc Surg. 2016, 31:252-5.

2. Sankar NP, Thakarar K, Rokas KE: Candida infective endocarditis during the infectious diseases and substance use disorder syndemic: a six-year case series. Open Forum Infect Dis. 2020, 7:ofaa142. 10.1093/ofid/ofaa142

3. Pierrotti LC, Baddour LM: Fungal endocarditis, 1995-2000. Chest. 2002, 122:302-10. 10.1378/chest.122.1.302

4. Rivoisy C, Vena A, Schaeffer L, et al.: Prosthetic valve Candida spp. endocarditis: new insights into longterm prognosis-the ESCAPE study. Clin Infect Dis. 2018, 66:825-32. 10.1093/cid/cix913

5. Jennifer M Boland, Heath H Chung, Frans J L Robberts, et al.: Fungal prosthetic valve endocarditis: Mayo Clinic experience with a clinicopathological analysis. Mycoses. 2011, 54:354-360. 10.1111/j.14390507.2010.01884.x

6. Silva-Pinto A, Ferraz R, Casanova J, Sarmento A, Santos L: Candida parapsilosis prosthetic valve endocarditis. Med Mycol Case Rep. 2015, 9:37-8. 10.1016/j.mmcr.2015.07.004

7. Pappas PG, Kauffman CA, Andes DR, et al.: Clinical practice guideline for the management of Candidiasis: 2016 update by the Infectious Diseases Society of America. Clin Infect Dis. 2016, 62:e1-50. 10.1093/cid/civ933

8. Steinbach WJ, Perfect JR, Cabell CH, et al.: A meta-analysis of medical versus surgical therapy for Candida endocarditis. J Infect. 2005, 51:230-47. 10.1016/j.jinf.2004.10.016 